\title{
CLINICAL SNAPSHOT
}

\section{Multiple Pulmonary Emboli of Bone Cement after Kyphoplasty}
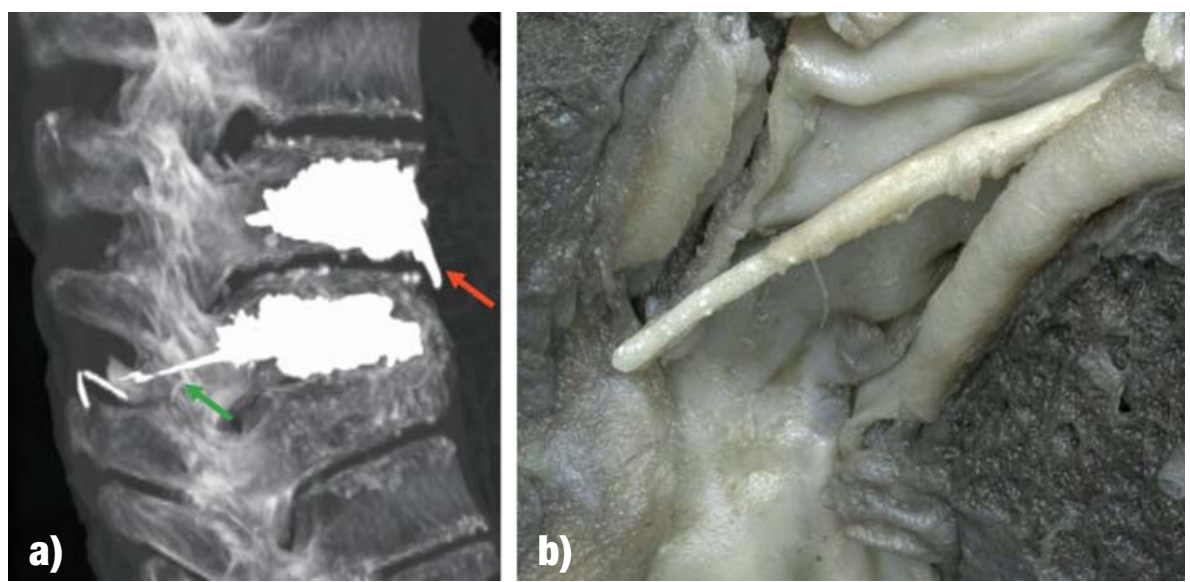

Figure

a) Local leakage of bone cement into the prevertebral venous plexus (red arrow), retrograde flow via the transpedicular drilled canal (green arrow). b) Cement embolus into a pulmonary artery, diameter $1 \mathrm{~mm}$.
We report the autopsy findings of a 75-year-old woman who died of an acute pulmonary arterial embolism secondary to deep venous thrombosis of the lower limbs. Computerized tomography had shown local leakage of cement from a kyphoplasty (T11, T12) performed three years earlier. Gross pathological examination of the lungs revealed needle-shaped cement emboli (Figure), and histopathological examination revealed multiple microemboli of bone cement, some of which were integrated into vessel walls. These findings demonstrate a rarely reported complication of this commonly performed surgical procedure. The clinical significance of pulmonary microemboli of bone cement may be underestimated; its full extent should be determined in future morphological studies. Already at present, kyphoplasty should only be performed when strictly indicated, and preventive measures against cement embolism must be taken. These include using cement of suitable viscosity, avoiding excessive pressure, and paying due consideration to the local vascular anatomy in the light of the patient's underlying disease.

Dr. med. Sebastian Butscheidt, Institut für Osteologie und Biomechanik, Universitätsklinikum Hamburg-Eppendorf, Hamburg, s.butscheidt@uke.de Jacob Ritter, Prof. Dr. med. Klaus Püschel, Institut für Rechtsmedizin, Universitätsklinikum Hamburg-Eppendorf

\section{Conflict of interest statement: The authors state that they have no conflict of interest.}

Cite this as: Butscheidt S, Ritter J, Püschel K: Multiple pulmonary emboli of bone cement after kyphoplasty. Dtsch Arztebl Int 2017; 114: 756. DOI: 10.3238/arztebl.2017.0756

Translated from the original German by Ethan Taub, MD 